bioRxiv preprint doi: https://doi.org/10.1101/2020.07.23.217547; this version posted July 24, 2020. The copyright holder for this preprint (which was not certified by peer review) is the author/funder, who has granted bioRxiv a license to display the preprint in perpetuity. It is made available under aCC-BY-NC-ND 4.0 International license.

\title{
The Latency of Spontaneous Eye Blinks Marks Relevant Visual and Auditory Information Processing
}

\author{
Supriya Murali ${ }^{1}$, Barbara Händel ${ }^{1}$ \\ ${ }^{1}$ Department of Psychology III, University of Würzburg
}

\section{* Corresponding author:}

Supriya Murali

supriya.murali@uni-wuerzburg.de 


\begin{abstract}
Eye blinks are influenced by several external sensory and internal cognitive factors. However, neither the precise temporal effects of these factors on blinking nor how their timing compares between modalities is known. Our aim was to understand the influence of sensory input vs task-relevant information on blinks in the visual and auditory domain.

Using a visual and an auditory temporal judgement task, we found that blinks were suppressed during stimulus presentation in both domains and the overall input length had a significant positive relationship with blink latency i.e. the end of blink suppression. Indeed, the effect of sensory input duration on blink latency was not significantly different between visual and auditory stimuli. The precise timing of blink latency was further modulated by the duration of the task relevant input, which was independent of the overall length of sensory input. The influence of task related changes embedded in the overall stimulation suggests an additional influence of top-down processes on blink timing. Intriguingly, embedded changes as short as $40 \mathrm{~ms}$ in the auditory domain and $100 \mathrm{~ms}$ in the visual domain are reflected in blink latency differences. Importantly, we could show that task accuracy and motor response was not the driving factor of blink modulation.

Our results show a sensory domain independent modulation of blink latencies introduced by changes in the length of task-relevant information. Therefore, blinks not only mark the time of sensory input but also can act as precise indicator for periods of cognitive processing and attention.
\end{abstract}

Keywords: Eye Blink, Blink latency, Blink suppression, Visual modality, Auditory Modality, Temporal judgment, Sensory influence, Cognitive influence, Attention 


\section{Introduction}

Humans generate about 12 spontaneous eye blinks in a minute (Fatt and Weissman 1992). While one main function is to moisten the corneal tear film, the frequency of their occurrence is much higher than what would be required for that purpose (Zametkin, Stevens and Pittman 1979). Three factors have been suggested to additionally influence the blink rate: sensory input processing, the internal mental state and motor output (Bonneh, Adini and Polat 2016, Cong, et al. 2010, Hoppe, Helfmann and Rothkopf 2018, Oh, Jeong and Jeong 2012, Siegle, Ichikawa and Steinhauer 2008, Wascher, et al. 2015)

Interestingly, not only the rate of blinks is affected by these factors, but also the blink timing. Instead of being randomly executed during a task, blinks follow a specific decreaseincrease-baseline pattern, which, Bonneh, Adini, \& Polat (2016), refer to as an "oculomotor inhibition mechanism". This pattern is characterized by decreasing or suppressing one's blinks during stimulus or information presentation and increasing it during or after the offset of that information (Hoppe, Helfmann and Rothkopf 2018, Oh, Jeong and Jeong 2012, Ohdra 1995, Siegle, Ichikawa and Steinhauer 2008). The purpose of reduced blinking, specifically during visual stimulus presentation, could be simply to not lose any incoming information, which is also a probable reason why visual tasks tend to have a lower blink rate (Goldstein, et al. 1985). However, the decrease is not specific to the visual modality but has been observed during auditory tasks as well (Bauer, et al. 1985, Kobald, et al. 2019) and the overall duration of a stimulus has been found to increase the duration of blink suppression in both domains (Bauer, et al. 1985, Oh, Jeong and Jeong 2012). This suggests a domain independent mechanism. A study by Hoppe, Helfmann, \& Rothkopf (2018) even showed that a mere expectation of a visual stimulus can have an influence on one's blinking which would mean that sensory input is not the driving factor of blink modulation, but that more general cognitive processes influence blinking. In fact, it has been argued that the decrease occurs during periods of attention allocation and that the increase represents the point when all information processing has been completed (Wascher, et al. 2015). This fits to the finding that the suppression of blinks is stronger for more difficult tasks (Oh, Jeong and Jeong 2012).

Apart from the factors pertaining to the stimulus or task itself, blinks have also been shown to be influenced by the motor response. A study by Colzato, van Wouwe, \& Hommel (2007) showed that blink rates can reflect the strength of visuomotor binding between a task relevant visual stimulus and the response involving a left and right key press. It has further been shown that blinks are modulated around the motor output (Bauer, et al. 1985, Goldstein, 
Bauer and Stern 1992, Oh, Han, et al. 2012) and can be entrained to the motor response even when participants engage in self-paced rhythmic finger tapping without external sensory cues (Cong, et al. 2010). Nevertheless, motor aspects seem not to be the sole influence on blink occurrence since blinks have been shown to be modulated even during no-go trials (Kobald, et al. 2019, Wascher, et al. 2015).

We wanted to understand and dissociate the influences of motor response, sensory input and cognitive processes on blink behavior and rigorously compare it between sensory domains. To this end, we independently manipulated task irrelevant and task relevant sensory input during a comparable auditory and visual task, and considered the motor output as well as more cognitive influences related to the task performance. We specifically focused on the temporal effects on blinking behavior by looking at the blink latencies, a measure which has been used previously to detect influences on blinking (Doughty 2001, Fogarty and Stern 1989, Goldstein, et al. 1985, Goldstein, Bauer and Stern 1992, Kobald, et al. 2019).

We conducted a visual and auditory temporal judgement task in which subjects had to judge if two stimuli appeared simultaneously. To secure comparable performance between the two modalities smaller time differences between the two stimuli were used in the auditory task, since temporal processing has been shown to be better for auditory input (Kanabus, et al. 2002). Also, since low level stimulus features can effect blinking (Bonneh, Adini and Polat 2016), we conducted the experiments in complete darkness. Our results showed that blinks were suppressed similarly in the two domains and that the length of this suppression correlated with the duration of the task-relevant information periods independent of the overall stimulus length or the motor response. Hence, our findings suggest a temporally precise domain general topdown modulation of eye blinks.

\section{$2 \quad$ Methods}

Eighteen subjects (4 males) aged between 18-35 years participated in the study. All participants gave their written informed consent and received either payment or study credit for their participation. The study was approved by the local ethics committee and was in line with the European general data protection regulations (DSVGO).

Two subjects were excluded for the visual condition because of an overall response accuracy below $10 \%$ and a mean blink rate of below 1 blink per minute respectively and one subject was excluded in the auditory condition for a low blink rate. Six additional subjects were 
excluded for the analysis pertaining to the ISI and Stimulus ON-time (i.e. duration until both stimuli were turned off) in the auditory condition, due to incomplete data recordings.

The mobile SMI Eye tracker glasses (ETG 2W Analysis Pro- 120Hz) was used to record eye data and responses were given via two response buttons connected to a response box (model: K-RB1-4; The Black Box ToolKit Ltd, UK), which was connected to a Dell Precision (M6700) laptop via a USB cable. The experiment and the analysis were coded in MATLAB 2012 and 2015a using the Psychtoolbox extensions (Brainard 1997, Kleiner, Brainard and Pelli 2007, Pelli 1997). All data streams were recorded using the Lab Streaming Layer, LSL (https://github.com/sccn/labstreaminglayer) along with LabRecorder (version 1.12b). The experiment was conducted in a completely dark room.

\subsection{Visual Condition}

The visual stimulus was presented using three red light emitting diodes (LEDs) with a diameter of $4 \mathrm{~mm}$, placed on a metal ruler using magnets. The LEDs were placed in front of the subject at a distance of $50 \mathrm{~cm}$ at eye level in a completely dark room (modified EEG booth). The central LED was only turned on at the start of the experiment and was switched off during the trials. The other two LEDs were placed on either hemifield, each at 11 degrees from the central fixation. During each trial, the two stimuli (LEDs) turned on at the same or at different times with inter-stimulus intervals (ISI) ranging from 0 to $0.2 \mathrm{~s}$ in steps of $0.02 \mathrm{~s}$. The 0 ISI indicates that both stimuli turn on simultaneously. The order of the stimuli (for ISI $>0$ ) was randomized within subjects. After their onset, the two stimuli remained on for $0.4,0.5$ or $0.6 \mathrm{~s}$, which is referred to as the stimulus ON-time (Duration both stimuli were on until the time both were turned off). Also this time period was randomly assigned per trial. After this, both LEDs were turned off. To further add to the unpredictability of the next trial, we jittered the next stimulus onset by randomly adding an inter-trial interval between 0.5 and $0.6 \mathrm{~s}$ (see Figure 1a). The participants had to indicate if the two lights turned on at the same or at different times by pressing either a left or a right button (randomly assigned for each subject) as fast as possible at any time during the trial. There were two sessions of 506 trials each, adding to a total of 1012 trials. We included one long break in between the sessions and two short breaks within each session to avoid excessive fatigue, which can be introduced by sitting in complete darkness.

\subsection{Auditory Condition}

The auditory condition was similar in structure and task but now the stimulus consisted of two sound clicks $(500 \mathrm{~Hz})$ that were presented in each ear using the Sennheiser PC3 headset 
along with the Steinberg UR22mk II external soundcard, again in complete darkness. The procedure and task was similar to the visual condition (see Figure 1b), with three differences: 1) the ISIs did not have an influence on the trial length i.e. the stimulus ON-time refers to the duration at least one stimulus was on until the time both were turned off) 2) The ISIs ranged from 0 to $0.1 \mathrm{~s}$ in steps of $0.01 \mathrm{~s}$. The lower ISI range for auditory was used since auditory discrimination thresholds have been found to be lower (see Introduction) 3) There was only one session of 506 trials, whereas the visual condition had two sessions. The reason for using two sessions in the visual condition was to obtain enough blinks, since blink rates are generally lower during visual tasks (see Introduction).

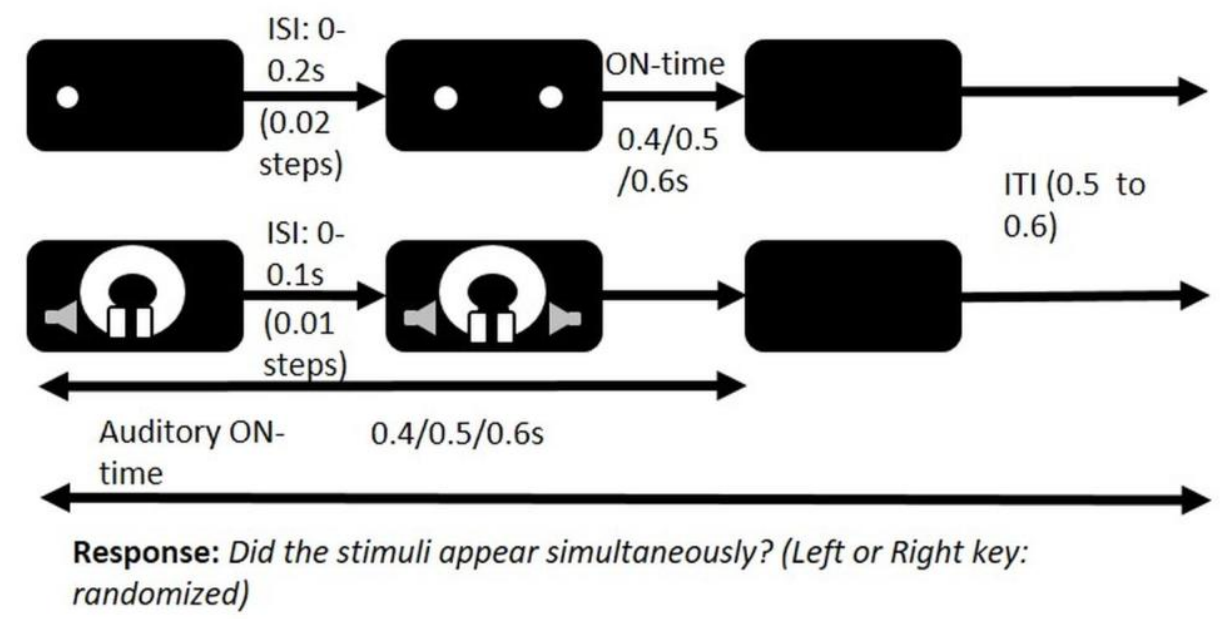

Figure 1. Stimulus and Task during the visual and the auditory conditions

\subsection{Stimulus Timing Test}

Before we started with data collection, we tested our stimulus to make sure that the timing was precise. This was done using a photodiode for the visual and an audio output recorder that measured the audio output directly from the audio jack.

\section{$3 \quad$ Analysis}

\subsection{Blink Detection}

We computed a blink detection algorithm based on the pupil radius data. The $\mathrm{z}$ value of the radius data was calculated and a potential blink was considered if either 1) both radii values were missing or 2) if either the left or right eye data was missing and the other eye had a $\mathrm{z}$ value below a threshold $(-1,-2$ or -3 , set individually because of considerable difference in signal to noise ratio in the data). 
The onset and the offset of the blink were computed based on the last point, for either eye, where the z-values was still above the threshold before the blink, or the first $\mathrm{z}$-value that reached above the threshold after the blink. Blinks with less than $0.016 \mathrm{~s}$ between them were combined and those with durations lower than $0.05 \mathrm{~s}$ or higher than $0.5 \mathrm{~s}$ were discarded.

\subsection{Blink rates (time resolved)}

The plotted time series consists of 0.1s non-overlapping time windows. We calculated the normalized mean number of blinks in each time window by dividing the mean number of blinks in that time window by the mean number of blinks in all time windows.

\subsection{Blink latency}

We also looked at three factors that could modulate the timing of blink occurrence: the stimulus ON-time (time until both stimuli were turned off), the ISI and the reaction time. We took the mean blink latency as the dependent measure (discussed in the next part).

For the factor ON-time, the blink latency was calculated as the difference between the first blink onset and the onset of the second stimulus i.e. discarding the influence of the ISI period. To account for the possibility that merely taking the overall average for each ON-time might lead to differences due to different number of blinks for each ISI within each ON-time, we first calculated the mean over each ISI within the ON-time and then averaged over these ISI specific means. This was followed by a two-factor ANOVA with factors ON-time $(0.4,0.5$, 0.6) and Modality (Visual vs. Auditory) and the mean Blink latency as the dependent variable.

For the factor ISI, a regression analysis was conducted. The dependent variable, the blink latency, was taken as the difference between the first blink onset and the trial onset. We followed the same procedure as describe above for the $\mathrm{ON}$-time, by calculating the mean over each ISI within each ON-time condition first and then calculated an overall mean.

Lastly, for the reaction time, we first calculated the mean blink latency from the trial onset and the mean reaction time (also from the trial onset) for each subject. A regression analysis with the reaction time as the predictor and blink latency as the dependent variable was then conducted. 


\section{$4 \quad$ Results}

\subsection{Overall Performance (accuracy and reaction time)}

The overall mean accuracy and reaction time for the visual condition was $48.5 \%(S D=$ $15.4 \%)$ and $0.56 \mathrm{~s}(S D=0.22 \mathrm{~s})$ respectively; and for the auditory condition $63.8 \%(S D=22.5 \%)$ and $0.5 \mathrm{~s}(S D=0.14 \mathrm{~s})$ respectively. For both the visual and the auditory conditions, the accuracy was highest for 0 ISI since it was easy to perceive the LEDs as lighting up or the tones appearing simultaneously. As further expected, the accuracy increased with increasing ISIs (for ISIs above 0) (Figure 2a and b). Regression analysis showed a significant linear relationship between accuracy and ISI (calculated from $0.02 \mathrm{~s}$ for the visual and from $0.01 \mathrm{~s}$ for the auditory condition) for both the visual $\left(R^{2}=0.98, b=0.02, F(1,8)=361.1, p<.001\right)$ and the auditory $\left(R^{2}=0.90\right.$, $b=0.4, F(1,8)=72.4, p<.001)$ condition.
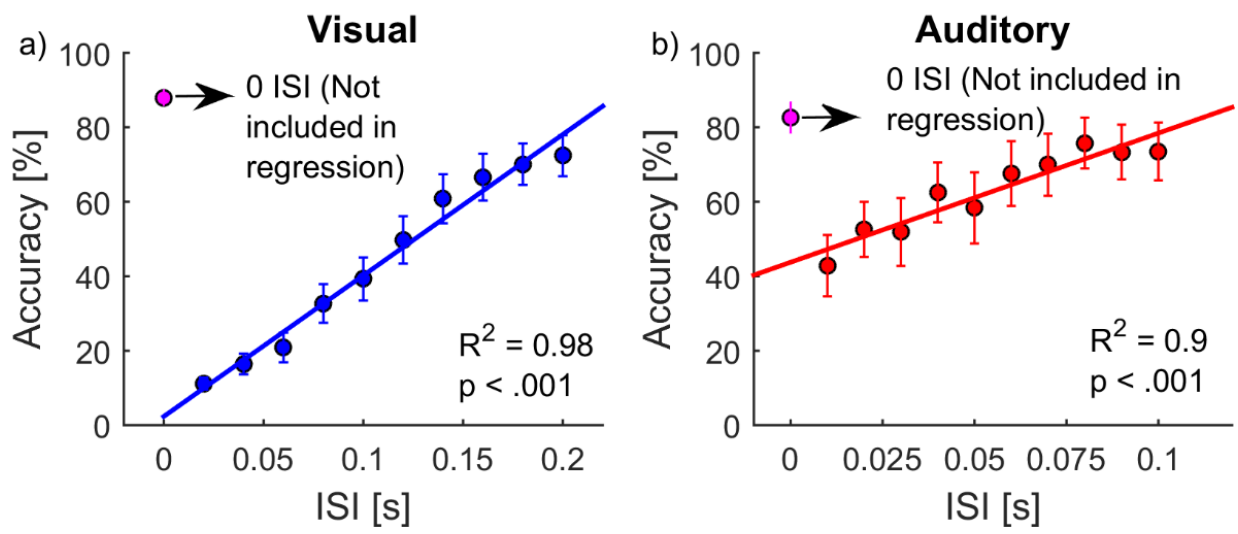

Figure 2. Interstimulus interval (ISI) vs. Accuracy. a) During the visual condition b) During the auditory condition. Error bars represent standard errors.

\subsection{General Blink Parameters (overall rate and duration)}

The mean blink rate during the visual condition was 7.8 per minute $(S E=2.02)$ and for the auditory it was 11.5 per minute $(S E=2.7)$, as shown in Figure 3. T-test showed a significant difference between the blink rates $(t(14)=2.6, p=.02)$. The mean duration of blinks was $0.14 \mathrm{~s}$ $(S E=0.01$ for the visual and $0.15 \mathrm{~s}(\mathrm{SE}=0.02)$ for the auditory conditions. 


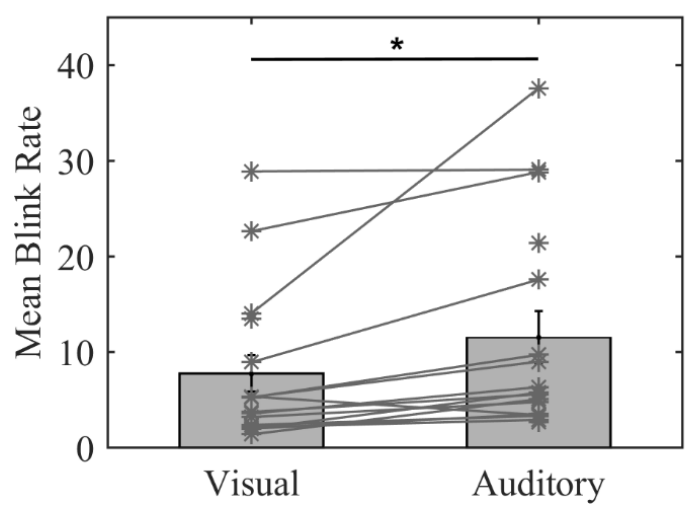

Figure 3. Mean blink rates during the visual and the auditory conditions. Error bars represent standard errors.

\subsection{Time Resolved Blink Rates}

Special interest was placed on the modulation of the blink rate over time within a trial. Specifically, we looked at the mean blink rate from $0.2 \mathrm{~s}$ before the trial onset to $2 \mathrm{~s}$ after the trial onset for both the modalities (Figure 4). The purpose was to see if we can replicate the previously described modulation in the visual domain and if it similarly occurs in the auditory domain. The graphs show a similar modulation in both modalities with an increase in blink rate starting about $0.3 \mathrm{~s}$ lasting until $1.2 \mathrm{~s}$ after the trial onset.

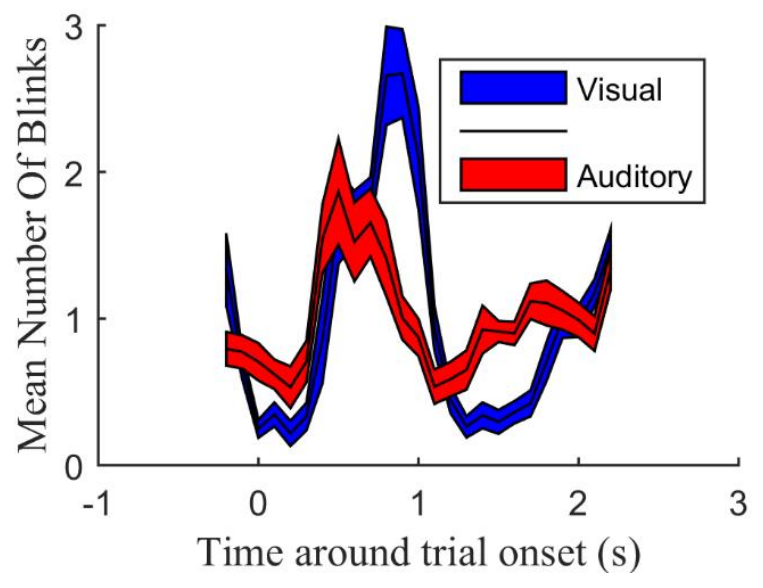

Figure 4. Normalized mean number of blinks during the visual and the auditory condition. The $\mathrm{x}$-axis represents timing with $0.1 \mathrm{~s}$ non-overlapping windows. The $\mathrm{y}$-axis represents the normalized mean number of blinks in each window over all subjects. The colored region represents the standard error. 


\subsection{Blink Latency}

In order to understand what influences the timing of this modulation, we looked at the latency of the first blink during each trial. Specifically, we investigated the possible influence of the task-independent sensory input defined by the ON-time (Time until the stimuli were turned off), the task-related input (ISI) and the timing of the motor response reflected in the reaction time.

4.4.1 Factor 1- Stimulus ON-time (time until both stimuli were turned off): (Taskindependent sensory input)

Figure 5 shows the mean blink latency for the three ON-times in the visual (blue bars) and auditory (red bars) conditions. A two factor repeated measures ANOVAs with factors Modality and ON-time, revealed only a significant effect of ON-time on the blink latency $(F$ $(2,61)=7.8, p=.001)$ and no significance for modality $(\mathrm{F}<1)$.

PostHoc t-tests revealed, for the visual condition, a significant difference between $0.4 \mathrm{~s}$ $(M=.6, S E=.02)$ and $0.6 \mathrm{~s}(M=.73, S E=.03)$ ON-time $(t(15)=3.2, p=.005)$; but not the between the 0.4 and $0.5 \mathrm{~s}$ ON-time $(t(15)=1.8, p=.09)$ and the $0.5 \mathrm{~s}(M=.68, S E=.03)$ and 0.6 s ON-time $(t(15)=2.1, p=.05)$. Similarly, for the auditory condition, the tests showed a significant difference between $0.4 \mathrm{~s}(M=.6, S E=.03)$ and $0.6 \mathrm{~s}(M=.7, S E=.03)$ ON-time $(t$ $(10)=3.6, p=.004)$; but not between $0.4 \mathrm{~s}$ and $0.5 \mathrm{~s}(M=.6, \mathrm{SE}=.03) \mathrm{ON}$-time $(t(10)=2.02$, $p=.07)$ and $0.5 \mathrm{~s}$ and $0.6 \mathrm{~s} \mathrm{ON}$-time $(t(10)=1.5, p=.16)$. Longer stimulus ON-time therefore lead to a longer blink latency. 


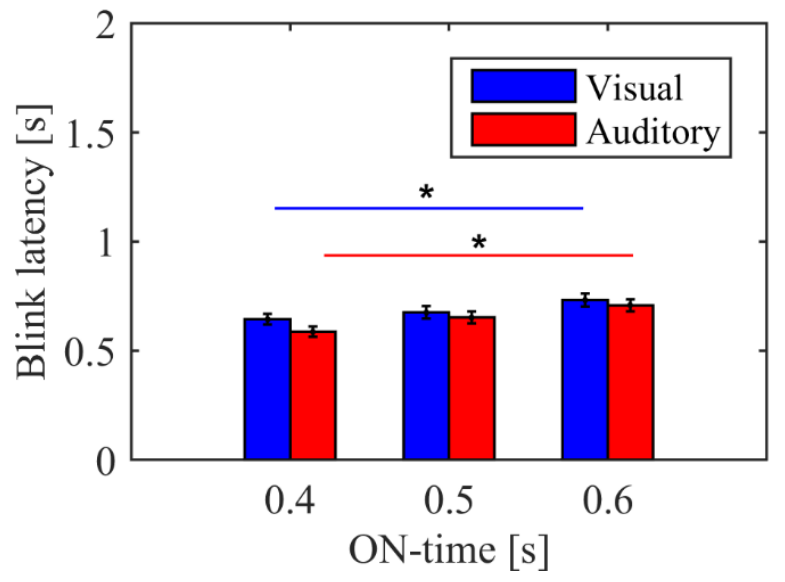

Figure 5. Comparison of blink latencies between the three ON-times (time until both stimuli were turned off) for the visual (blue) and the auditory (red) condition. The error bars represent standard error. Asterix marks significant differences at the level of 0.05 alpha based on a post-hoc Ttest.

\subsubsection{Factor 2: ISI (Task- dependent input)}

Increasing ISIs denoted increasing input durations of task relevant information. However, ISIs also positively correlate with accuracy, which could lead to cognitive influences. We therefore needed to dissociate accuracy effects from input duration effects. As shown in Fig 2, the ISIs showed a positive relationship with accuracy with the exception of the synchronous onset (0 ISI), which showed accuracies comparable to the highest ISI (Figure $2 \mathrm{a}$ and b). Hence, if the effect of the ISI is due to the increase in input duration, the synchronous onset would follow a positive correlation with blink latency. However, if it is due to accuracy effects, the 0 and the highest ISIs would have comparable latencies (since they led to similar accuracy).

As a first step, we looked at whether there is an effect of the ISI, without considering the 0 ISI, for the visual and the auditory condition respectively (Figure $6 a$ and $b$ ). The regression analysis showed that the ISI significantly predicted the blink latency for both the visual $\left(R^{2}=\right.$ $.46, b=.731, F(1,8)=6.98, p=.03)$ and the auditory $\left(R^{2}=.41, b=.64, \mathrm{~F}(1,8)=5.65, p=.04\right)$ condition. Please note that for the auditory condition, the ISIs did not lead to an overall change in stimulus length.

We then compared the 0 ISI with the highest ISI in both conditions (also shown in the same Figure $6 \mathrm{a}$ and $\mathrm{b}$ ). For the visual task (Figure 6a, we found a significant difference $(t(15)$ $=3.8, p=.002)$ between the 0 ISI $(M=.73, S E=0.02)$ and the 0.2 ISI $(M=.85, S E=0.035$. However, for the auditory condition (Figure 6b), we found no significant difference $(t(10)=$ $.4, p=.705)$ between the 0 ISI $(M=.68, S E=.09)$ and the 0.1 ISI $(M=.72, S E=.03)$. 

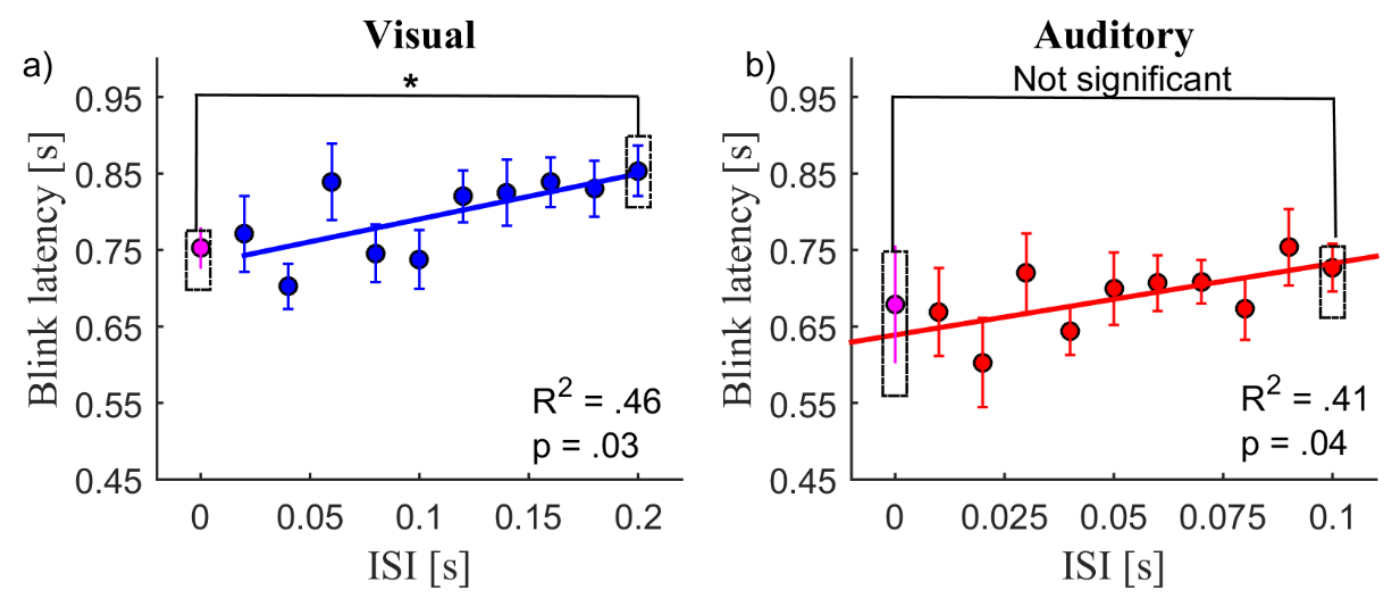

Figure 6. ISI vs Blink latency. Regression analysis did not include the 0 ISI. Ttest (represented in dotted lines) conducted between the 0 ISI and the highest ISI $(0.2 \mathrm{~s}$ for the visual and $0.1 \mathrm{~s}$ for the auditory). Error bars represent standard errors.

Additionally, to see how the increase in ISI leads to increase in blink latency, we plotted a psychometric function (Figure 7) based on the data from Figure 6. Specifically, for the visual task, we compared ISIs that were $0.02 \mathrm{~s}$ upto $0.140 \mathrm{~s}$ (in steps of $0.02 \mathrm{~s}$ ) apart. For the auditory task we used 0.01 to $0.07 \mathrm{~s}$ ISI difference (in steps of $0.01 \mathrm{~s}$ ). For instance, a difference in ISI of $0.04 \mathrm{~s}$, would mean that we compare data (blink latency) for the ISI of 0.02 to the one for ISI 0.6, 0.04 to 0.8 and so on untill 0.16 to 0.2 . We then calculated how many of these pairs showed an increase in blink latency. This results in a percentage of data points showing a positive change in blink latency dependent on how large the time difference was between the ISIs. Note that, since the number of available data points decrease as we increase the difference in ISI, we refrain from statistics but add a psychometric function for description of the trend. Looking at Figure 7, the function suggests that a change in ISI of $0.1 \mathrm{~s}$ for visual and $0.04 \mathrm{~s}$ for auditory leads to an increase in blink latency $80 \%$ of the time. 


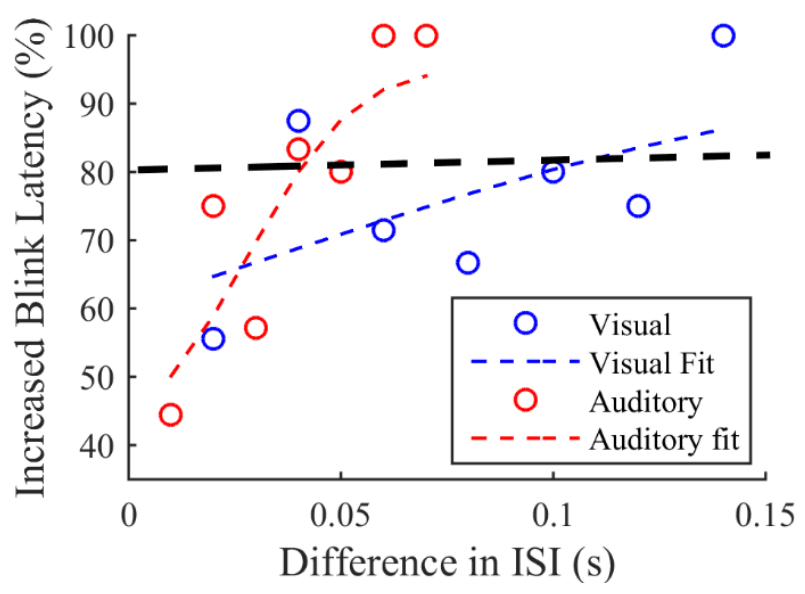

Figure 7. The percentage of cases (including a psychometric fit) in which an increase of ISI of a certain length led to a concurrent increase in blink latency. The x-axis shows the difference between the ISIs that are taken to compare the blink latency (as taken from Figure 6). The y-axis shows the percentage of data points were in an increase in ISI (as specified on the $\mathrm{x}$-axis) led to an increase in blink latency

\subsubsection{Factor 3: Reaction time}

Figures $8 \mathrm{a}$ and $\mathrm{b}$ show the relationship between the reaction time and the blink latency. The regression analysis showed no significant influence of the RT in either of the conditions (visual: $R^{2}=.11, b=1.1, F(1,14)=1.65, p=.22 ;$ auditory: $\left.R^{2}=.11, b=0.46, F(1,15)=1.92, p=.2\right)$ and additionally shows a reversed slope for the auditory and visual condition.
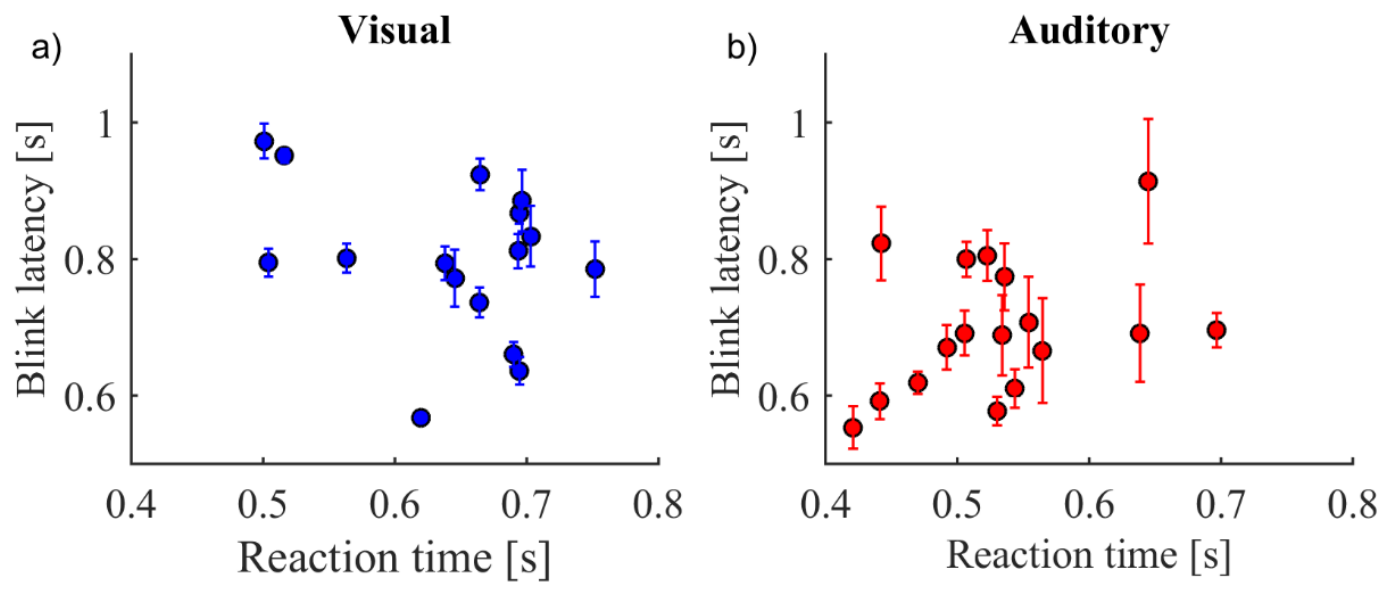

Figure 8. Regression analysis between the Reaction time and Blink Latency the visual (a) and the auditory (b) 


\section{$5 \quad$ Discussion}

Using an auditory and visual simultaneity judgment task, our study shows a temporally precise modulation of blink latency influenced by sensory as well as cognitive factors, similarly in the auditory and visual domain. Specifically, periods involving stimulus presentation were associated with a low blink rate and an increase after its offset. Our aim was to understand what influences this modulation by investigating the duration of the period of reduced blinking during stimulus presentation by looking at the timing of the first blink after the onset of the stimulus, referred to as blink latency. This time can be interpreted as period of blink suppression. We specifically looked at the influence of the following factors on the blink latency: the overall sensory input, the time of the manual response (reaction time), the task specific sensory input (ISI) and indirectly at task accuracy. A rigorous comparison between these different factors in a comparable visual and auditory task is missing to date.

The overall sensory input duration showed the most robust effect, in the sense that, people always tended to wait until the offset of the sensory input before starting to blink. Figure 5 shows that the blink latency was approximately 0.6 s for a stimulus ON-time (time until both stimuli were turned off) of $0.4 \mathrm{~s}$. Note that, the latency was calculated from the time both stimuli were turned on, which was the beginning of the $0.4 \mathrm{~s}$ period and the end of this period marks the stimulus offset. Hence, a blink was most likely to occur after the stimulus was turned off. The pattern of reduced blink rate followed by an increase has been reported before in both the visual (Oh, Jeong and Jeong 2012, Siegle, Ichikawa and Steinhauer 2008) as well as the auditory (Oh, Han, et al. 2012, Oh, Jeong and Jeong 2012) domains. It was interpreted as an active suppression followed by its rebound (Hoppe, Helfmann and Rothkopf 2018, Oh, Jeong and Jeong 2012). In fact, it has been suggested that blinks follow the same inhibitory mechanism as other eye movements during the processing of any sensory event (Bonneh, Adini and Polat 2016).

In the visual domain, this suppression could be mediated by the desire to not lose any information; which is also why blink rates are usually lower for visual tasks (Goldstein R. W., 1985). However, similar to other researchers (Oh, Han, et al. 2012, Oh, Jeong and Jeong 2012), we also found the same modulation of blink latency in the auditory domain, which indicates that an interpretation based on visual input processing is insufficient. Indeed, it has been argued that the decrease in blinks is associated with attention to the input, where the subsequent increase in blinks marks the end of the attentional period i.e. when all information processing 
is complete (Kobald, et al. 2019, Wascher, et al. 2015). The suppression of blinks until its release is possibly a result of attentional allocation to sensory input. In fact, a study by Hoppe, Helfmann, \& Rothkopf (2018) showed that even a mere increase in the probability of a visual event was sufficient to lower the probability of blinks, strengthening the idea of a non-sensory driven active top-down influence. Therefore, a precise temporal modulation in blinks through top-down factors could occur independent of the sensory domain and sensory input.

Indeed, our further findings suggest such a domain general attentional effect in blink suppression independent of the overall sensory input duration. We found that task-specific sensory input (ISI), embedded in the overall sensory input, was significantly associated with increasing blink latencies (Figure $6 \mathrm{a} \& \mathrm{~b}$ ). Subjects still waited on average until the overall stimulation offset, but the length of the task-relevant (to be attended) input added time to the sensory based (bottom-up driven) latency. Please note that we argue here based on the auditory results, since in the visual task, the ISI added to the overall input length (Figure 1). However, since the main effects of the different ON-times (time until both stimuli were turned off) were comparable between the two modalities, this difference might be negligible. Assuming that subjects wait until both LEDs/ tones have been presented before deciding if they turned on simultaneously or not, the ISI increased the duration the relevant input needed to be complete. This would increase the length of attended time within the overall sensory input. Our data (excluding simultaneous onset) showed a strong positive correlation with blink latency. Increased ISI however is also highly correlated with accuracy. Accuracy increases with the increase in ISI (Figure 2a and b), except (not surprisingly) for the 0 ISI which is simple to detect as simultaneous input. This specific case helps to understand that it is not accuracy related effects that drive the blink latency, but indeed the length of task relevant input. Nevertheless, there might still be an influence of task difficulty as shown by (Drew 1951, Goldstein, Bauer and Stern 1992, Veltman and Gaillard 1998) which is masked by the influences of sensory input. We argue against a role of accuracy in explaining our findings in the following paragraph.

While accuracy is similarly high for 0 ISI in both modalities, the time of relevant input however is different for the two modalities. This stems from a basic perceptual difference between the modalities. For visual input, two spatially non-overlapping but temporally overlapping signals can be perceived easily as two separate inputs, whereas this is not the case for a temporally overlapping tone presented to the left and right ear. Two signals that are sufficiently correlated between the ears are fused and interpreted as a single auditory event (Blauert 1938). This is known as binaural fusion and has been widely studied (Broadbent 1955, Leakey 1958, Masakazu 1991). In our task, this fusion forces subjects to wait until the 
probability of the second tone to occurred is close to zero (which would be after the highest possible ISI period), before being able to decide what answer to give while the answer itself will be very accurate. Therefore, while accuracy for 0 ISI is similar for the auditory and visual task, the time attention needs to be focused on the input differs between modalities. This can explain why the 0 ISI in the auditory condition led to a blink latency that exceeded the one of the highest ISI while the 0 ISI in the visual task has the lowest blink latency. This finding clearly suggests that it is not accuracy related effects but the time of task relevant input (and likely its attention focused on it) which influences blink latency similarly in both sensory domains. Since task relevance is defined by the instruction and independent of the overall sensory input, a topdown influence on the blink latency can therefore be assumed.

Finally, our results show that the motor response or its planning played no part in the modulation of blink latency. Although, motor output can have an independent influence on blinking (Cong, et al. 2010, van Dam and van Ee 2006), our data (Figure 8a \& b) showed no correlation between the manual response and blink latencies.

In summary, while a decrease in blinks was previously associated with sensory input in the visual domain, we find the same modulation during purely auditory input. Additionally, our results show that minute changes of task relevant information length, even if embedded in ongoing sensory stimulation, modulate blinking behavior similarly in the auditory and visual domain (Figure 7a \& b). Our study therefore highlights domain general top-down influences that can precisely modulate the timing of blinking, mapping small temporal changes in sensoryattentional demands.

\section{Funding}

This project has been funded by the European Research Council (grant number 677819 awarded to B. Händel).

\section{References}

Colzato, Lorenza S, Nelleke C van Wouwe, and Bernhard Hommel. 2007. "Spontaneous eyeblink rate predicts the strength of visuomotor binding." Neuropsychologia 23872392.

Bauer, Lance O, Barbara D Strock, Robert Goldstein, John A Stern, and Larry C Walrath. 1985. "Auditory Discrimination and the Eye Blink." Psychophysiology 636-641.

Bentivoglio, Anna Rita, Susan B Bressman, Emanuele Cassetta, Donatella Carretta, Pietro Tonali, and Alberto Albanese. 1997. "Analysis of Blink Rate Patters in Normal Subjects." Movement Disorders 1028-1034. 
Blauert, J. 1938. "Psychoacoustic binaural phenomena." In HEARING_Physiological Bases and Psychophysics , 182-189. Berlin, Heidelberg: Springer.

Bonneh, Yoram S, Yael Adini, and Uri Polat. 2016. "Contrast sensitivity revealed by spontaneous eyeblinks: Evidence for a common mechanism of oculomotor inhibition." Journal of Vision 1-15.

Bonneh, Yoram, Moshe Fried, and Yael Adini. 2015. "Blinking by Surprise: Eye-Blink Rate and Latency Uncover Stimulus Predictability." Journal of Vision 779-779.

Brainard, D H. 1997. "The Psychophysics Toolbox." Spatial Vision 433-436.

Broadbent, D E. 1955. "A note on binaural fusion." Quarterly Journal of Experimental Psychology 46-47.

Chermahini, Soghra Akbari, and Bernhard Hommel. 2010. "The (b)link between creativity and dopamine: Spontaneous eye blink rates predict and dissociate divergent and convergent thinking." Cognition 458-465.

Cho, Pauline, Caren Sheng, Carol Chan, Raymond Lee, and Joey Tam. 2000. "Baseline blink rates and the effect of visual task difficulty and position of gaze." Current Eye Research 64-70.

Cong, D.K., M Sharikadze, G Staude, H Deubel, and W Wolf. 2010. "Spontaneous eye blinks are entrained by finger tapping." Hum Movem Sci 1-18.

De Jong, Peter J, and Harald Merckelbach. 1990. "Eyeblink Frequency, Rehearsal Activity, and." Harald Merckelbach 89-94.

Deubel, Heiner, Bruce Bridgeman, and Werner X Schneider. 2004. "Different effects of eyelid blinks and target blanking on saccadic suppression of displacement." Perception \& Psychophysics 772-778.

Doughty, Michael J. 2001. "Consideration of Three Types of Spontaneous Eyeblink Activity in Normal Humans: during Reading and Video Display Terminal Use, in Primary Gaze, and while in Conversation." Optometry and Vision Science 712-725.

Drew, G. C. 1951. "Variations in Reflex Blink-Rate during Visual-Motor Tasks." Quarterly Journal of Experimental Psychology 73-88.

Fatt, I, and B A Weissman . 1992. Physiology of the Eye: An Introduction to Vegetative Functions. Boston: Butterworth-Heinemann.

Fogarty, Christine , and A John Stern. 1989. "Eye movements and blinks: their relationship to higher cognitive processes." International Journal of Psychophysiology 35-42.

Gao , Xin, Hongmei Yan, and Hong-jin Sun. 2015 . "Modulation of microsaccade rate by task difficulty revealed through between- and within-trial comparisons." Journal of Vision $1-15$.

Golan, Tal, Shany Grossman, Leon Y Deouell, and Rafael Malach. 2018. "Widespread suppression of high-order visual cortex during blinks and external predictable visual interruptions." bioRxiv .

Goldstein, R, L C Walrath, J A Stern, and B D Strock. 1985. "Blink Activity in a Dicrimination Task as a Function of Stimulus Modality and Schedule of Presentation." Psychophysiology 629-635. 
bioRxiv preprint doi: https://doi.org/101101/2020 07 23.217547: this version posted July 24,2020 . The copyright holder for this preprin (which was not certified by peer review) is the author/funder, who has granted bioRxiv a license to display the preprint in perpetuity. It is made available under aCC-BY-NC-ND 4.0 International license.

Goldstein, Robert , Lance O Bauer, and John A Stern. 1992. "Effect of task difficulty and interstimulus interval on blink parameters." International Journal of Psychophysiology 111-117.

Higgins, Stephen J, David E Irwin, Ranxiao Frances Wang, and Laura E Thomas. 2009. "Visual direction constancy across eyeblinks." Attention, Perception, \& Psychophysics 1607-1617.

Holland, K K, and G Tarlow. 1972. "Blinking and mental load." Psychological Reports 119127.

Holland, M K, and G Tarlow. 1975. "Blinking and thinking." Perceptual and Motor Skills 503-506.

Hommel, Bernhard. 1998. "Event files: Evidence for automatic integration of stimulus response episodes." Visual Cognition 183-216.

Hoppe, David, Stefan Helfmann, and Constantin A Rothkopf. 2018. "Humans quickly learn to blink strategically in response." PNAS 2246-2251.

Ito, Junji , Andrey R Nikolaev, Marjolein Luman, Maartje F Aukes, Chie Nakatani, and Cees van Leeuwen. 2003. "Perceptual switching, eye movements, and the bus paradox." Perception 681-698.

Kanabus, Magdalena, Elzbieta Szelag, Ewa Rojek, and Ernst Poeppel. 2002. "Temporal order judgement for auditory and visual stimuli." Acta Neurobiology 263-270.

Kleiner , M, D Brainard , and D Pelli . 2007. "“What's new in Psychtoolbox-3?"." Perception 36 ECVP Abstract Supplement.

Kobald, Oliver S, Edmund Wascher, Holger Heppner, and Stephan Getzmann. 2019. "Eye blinks are related to auditory information processing: evidence from a complex speech perception task." Psychological Research 1281-1291.

Leakey, D M. 1958. "Binaural Fusion of Low- and High-Frequency Sounds." The Journal of the Acoustical Society of America 222.

Masakazu, Konishi. 1991. "Neural mechanisms of binaural fusion." Modularity and the motor theory of speech perception 295-311.

Nakano, Tamami, and Shigeru Kitazawa. 2010. "Eyeblink entrainment at breakpoints of speech." Exp Brain Res 577-581.

Nakano, Tamami, Makoto Kato, Yusuke Morito, Seishi Itoi, and Shigeru Kitazawa. 2013. "Blink-related momentary activation of the default mode network while viewing videos." PNAS (2013) 702-706.

Oh, Jihoon, Mookyung Han, Bradley S Peterson, and Jaeseung Jeong. 2012. "Spontaneous Eyeblinks Are Correlated with Responses during the Stroop Task." PlosOne 1-11.

Oh, Jihoon, So-Yeong Jeong, and Jaeseung Jeong. 2012. "The timing and temporal patterns of eye blinking are dynamically modulated by attention." Human Movement Science 1353-1365.

Ohdra, Hideki . 1995. "Analysis of eyeblink activity during self-referent information processing in mild depression." Perceptual and Motor Skills. 
Pelli, D G. 1997. "The VideoToolbox software for visual psychophysics: Transforming numbers into movies." Spatial Vision 437-442.

Poulton, E. C. , and R. L. Gregory. 1952. "Blinking during visual tracking." Journal of Experimental Psychology 57-65.

Siegle, Greg J, Naho Ichikawa, and Stuart Steinhauer. 2008. "Blink before and after you think: Blinks occur prior to and following cognitive load indexed by pupillary responses." Psychophysiology 679-687.

Slagter, Heleen A, Katerina Georgopoulou, and Michael J Frank. 2015. "Spontaneous eye blink rate predicts learning from negative, but not." Neuropsychologia 126-132.

van Bochove, Marlies E, Lise Van der Haegen, Wim Notebaert, and Tom Verguts. 2013. "Blinking predicts enhanced cognitive control." Cogn Affect Behav Neurosci 346-354.

van Dam, Loes C.J. , and Raymond van Ee. 2006. "The role of saccades in exerting voluntary control in perceptual and binocular rivalry." Vision Research 787-799.

van Dam, Loes C.J., and Raymond van Ee. 2005. "The role of (micro)saccades and blinks in perceptual bi-stability from slant rivalry." Vision Research 2417-2435.

Veltman, J. A., and A.W. K. Gaillard. 1998. "Physiological workload reactions to increasing levels of task." Ergonomics 656 - 669.

Wang, Yanfang, Sonia S Toor, Ramesh Gautam, and David B Henson. 2011. "Blink Frequency and Duration during Perimetry and Their Relationship to Test-Retest Threshold Variability." IOVS, 4546-4550.

Wascher, Edmund, Holger Heppner, Tina Möckel, Sven Oliver Kobald, and Stephan Getzmann. 2015. "Eye-blinks in choice response tasks uncover hidden aspects of information processing." EXCLI journal 1207-1218.

Zametkin, A J, J R Stevens, and R Pittman. 1979. "Ontogeny of spontaneous blinking and of habituation of the blink reflex." Annals of Neurology 453-457. 